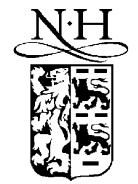

ELSEVIER

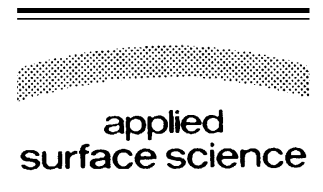

www.elsevier.com/locate/apsusc

\title{
Digital measurement of positron lifetime
}

\author{
K. Rytsölä*, J. Nissilä, J. Kokkonen, A. Laakso, R. Aavikko, K. Saarinen \\ Laboratory of Physics, Helsinki University of Technology, P.O. Box 1100, FIN-02015 HUT, Espoo, Finland
}

\begin{abstract}
A prototype of a digital positron lifetime apparatus is presented. We demonstrate that direct digitizing of detector pulses and subsequent simple analysis with software, can be used to replace the conventional analog electronics chain (constant-fraction discriminators, time-to-amplitude converter and multichannel analyzer). In this work, we use a fast digital oscilloscope. The quality of the lifetime data is shown to be as good as with a usual apparatus. For a pulsed positron lifetime beam the digital system is particularly suitable as no coincidence detection is needed and only one analog pulse has to be analyzed. (C) 2002 Elsevier Science B.V. All rights reserved.
\end{abstract}

PACS: 78.70.Bj; 07.85.Nc; 29.30.Kv

Keywords: Short time interval measurement; Fast coincidence apparatus; Positron lifetime spectrometer

\section{Introduction}

Positron lifetime spectroscopy has an established position in defect studies of solids [1]. During the last decades many important vacancy-type defects in bulk specimen have been unambiguously identified with the technique [1]. Currently, the method is extending to the studies of technologically important thin films as a result of the advent of various pulsed-beam concepts.

In bulk studies, the positron lifetime is usually measured as the time difference between two $\gamma$-quanta, the first one being emitted simultaneously with the positron from the source, and the second one being the annihilation quantum. In pulsed positron lifetime beam measurements, the start signal is obtained from the pulsing electronics and the stop signal again from the detection of an annihilation photon. Conventionally, the time difference between

\footnotetext{
${ }^{*}$ Corresponding author. Tel.: 358-9-451-3102.

E-mail address: klaus.rytsola@hut.fi (K. Rytsölä).
}

the start and stop signals is extracted using analog electronics: constant-fraction discriminators (CFD), time-to-amplitude converters (TAC) and multi-channel analyzers (MCA). Especially optimization of the CFD's has proven to be tedious. The whole electronics chain is also rather expensive.

In this paper, we present the first version of a digital positron lifetime spectrometer. We show that the replacement of the conventional analogue measurement electronics with a fast digitizer circuit is feasible. In this work we use a state-of-the-art digital oscilloscope to digitize the anode pulses from the detectors, after which the lifetimes are extracted from the sample sequence with software in a PC. A similar idea has recently been applied to time-of-flight measurements with silicon-strip detectors by Codino [2]. The performance of our apparatus is close to that achieved with an analog one, except for a rather low data throughput rate (15 counts per second) due to limitations in data transfer. This problem will be solved in the future by using a more sophisticated digitizing 
apparatus. The digital lifetime spectrometer is simple and easy to use, and is already now inexpensive compared to its conventional counterparts.

\section{Setup and methods}

Our detectors were composed of plastic $\phi 30 \times$ $20 \mathrm{~mm}^{3}$ cylindrical scintillators coupled to Philips/ Photonis XP2020photomultiplier tubes (PMT). The anode pulses from the start and stop detectors are combined and led to a digital oscilloscope (Tektronix TDS 3052, $5 \mathrm{GS} / \mathrm{s}, 500 \mathrm{MHz}$ ) which samples the pulses at 200 ps intervals (leading to about 100 samples per pulse). In the preliminary setup described in Fig. 1, a slow channel is still needed for triggering. This enhances the throughput of data by making sure that only coincident proper-sized pulses are handled. The sampled data are led via ethernet to a PC in which off-line analysis can be performed.

Fig. 2(a) shows an example of a digitized positron lifetime event. The left anode pulse originates from the $1275-\mathrm{keV} \gamma$-quantum from the ${ }^{22} \mathrm{Na}$ source and the right one from the annihilation photon. The positron lifetime is extracted from the pulse pair by calculating a cross-correlation with a smooth model pulse. From

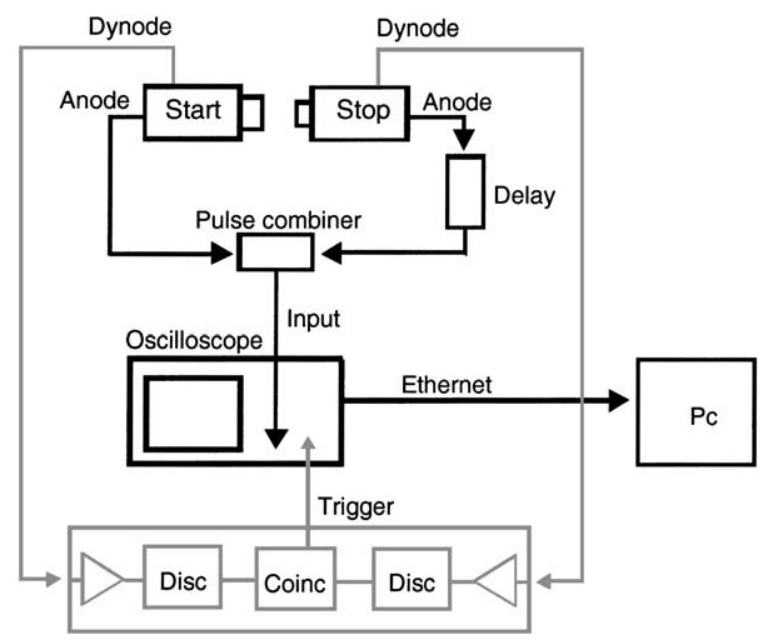

Fig. 1. Schematic diagram of a 'first generation' digital positron lifetime spectrometer.

the cross-correlation data (Fig. 2(b)) the lifetime is calculated simply as the difference between the locations of the peaks.

The energy windows, i.e. the pulse-height ranges of useful pulses, can easily be selected with software based on the amplitude spectra of the pulses in the lifetime data. This makes it possible to automatically

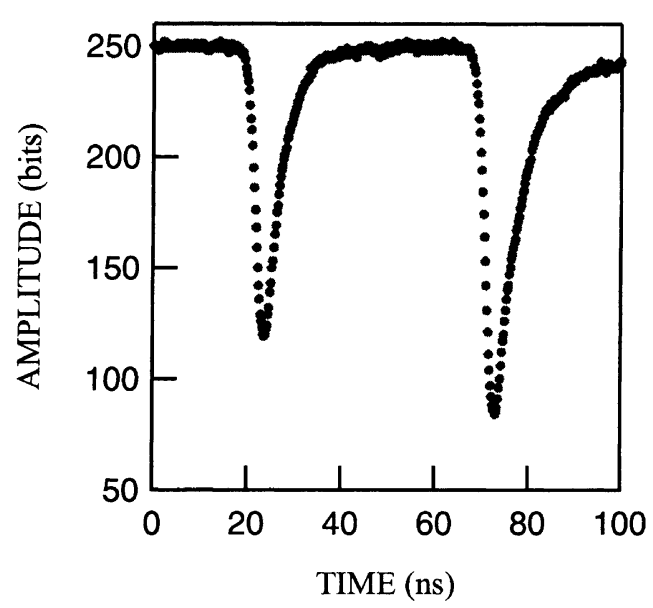

(a)

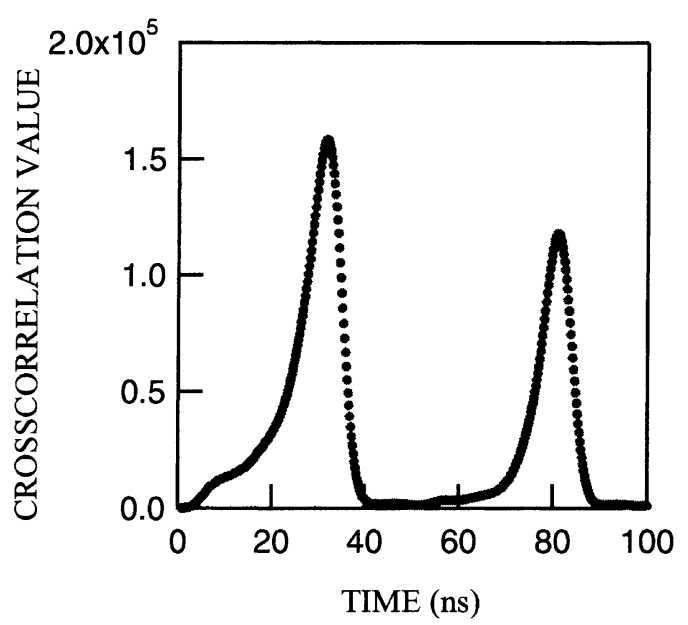

(b)

Fig. 2. (a) A typical digitized lifetime event captured by the oscilloscope. About 15 samples are collected within the rise time of the pulse. The timing information is extracted from the digitized pulses by crosscorrelation with a model pulse, the result of which is given in (b). The positron lifetime is then calculated as a time difference between the peak positions. 
take into account for example changes in the gain of the PMT during a long measurement series [3].

\section{Performance of the system}

To investigate the performance of the digital system we performed relevant test measurements. The 'electronic' resolution of the apparatus can be estimated by feeding the same anode pulse twice into the combiner. The observed time spread due to the A/D converter and the numerical timing algorithm is $33 \mathrm{ps}$ (full width at half maximum, FWHM) which corresponds to that of a combination of a conventional TAC and MCA. It is so small that its contribution to the overall resolution of the system is negligible. Also the linearity of the apparatus was found to be sufficient for positron lifetime measurements. It was tested with the aid of randomly distributed start pulses and also by the standard method based on the use of delay cables of accurately known lenghts.

The total resolution of our spectrometer was measured with a ${ }^{60} \mathrm{Co}$ source emitting two simultaneous $\gamma$-quanta. The result with 50\% Co windows was 203 ps

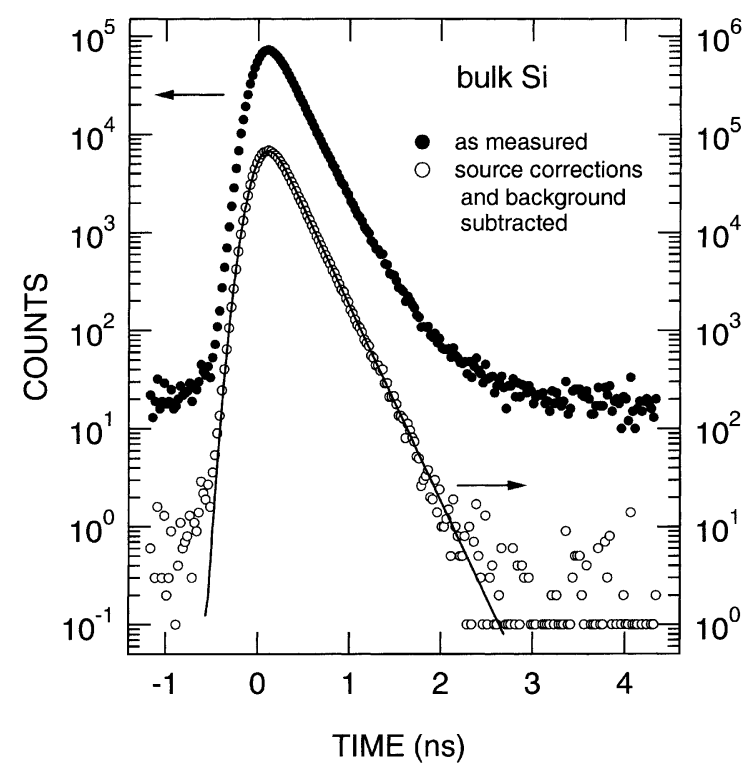

Fig. 3. Positron lifetime spectrum in bulk Si measured with a digital spectrometer. The solid line illustrates the best fit of a single exponential component convoluted with a Gaussian resolution function to the data from which source corrections and background have been subtracted.
(FWHM), which is a typical figure for a spectrometer with similar-sized scintillators.

Fig. 3 presents a positron lifetime spectrum measured in bulk $\mathrm{Si}$ with the digital apparatus. A single lifetime component of 218 ps was observed which is in perfect agreement with previous investigations [1]. In this measurement the time resolution was $297 \mathrm{ps.}$ Recently, preliminary tests with an improved version have already given a better resolution.

These studies show that positron lifetime measurements can be performed by sampling the detector pulses with a fast digital oscilloscope, and by extracting the lifetimes from the events numerically. The time resolution similar to that obtained with analog instruments indicates that the sampling rate of $5 \mathrm{GS} / \mathrm{s}$, and the overall quality of a fast A/D converter, are clearly adequate.

\section{Conclusions}

A 'first generation' digital lifetime spectrometer has been set up and tested. The performance is close to that typically obtained with analog pulse processing electronics.

The digital spectrometer has apparent advantages. Firstly, the system is simple, consisting only of the detectors and the digitizer. Hence, it is very easy to set up. The often difficult optimization of the timing discriminators is fully avoided. Moreover, the time stability and linearity are expected to be very good because of the crystal-controlled time axis. Since all the raw data can be stored in a computer, many exciting opportunities open due to the possibility of off-line analysis of individual lifetime events. For example, different timing algorithms are easy to investigate with a given set of pulse pairs. Various corrections on the data are also feasible, for instance pulse-amplitude related differences in lifetime spectrum position (walk) and residual drift of the spectrum due to the detectors can be eliminated. Furthermore, at least part of distorted pulses, e.g. due to pile-up, can be discarded.

Currently, we are about to implement a $2 \mathrm{GS} / \mathrm{s}$, $500 \mathrm{MHz}$ digitizer PCI-card which enables one to achieve nearly $100 \%$ data throughput. We are also searching for the optimum timing algorithm, focusing on the leading edge of the anode pulse. A similar system will also be used in the data collection of the 
pulsed positron lifetime beam under construction at Helsinki University of Technology. The digital measurement is particularly suitable to this purpose since even the small problems related to coincidence detection and continuous triggering by the pulsing frequency are avoided [4].

\section{Acknowledgements}

The discussions with Prof. P. Hautojärvi are gratefully acknowledged.

\section{References}

[1] A. Dupasquier, A.P. Mills, Jr. (Ed.), Positron Spectroscopy of Solids, IOS Press, Amsterdam, 1995;

K. Saarinen, P. Hautojärvi, C. Corbel, in: M. Stavola (Ed.), Identification of Defects in Semiconductors, Academic Press, New York, 1998;

R. Krause-Rehberg, H.S. Leipner, Positron Annihilation in Semiconductors, Springer, Heidelberg, 1999.

[2] A. Codino, Nucl. Instr. and Meth. A 440 (2000) 191.

[3] F. Bečvár̆, J. Čížek, L. Lešták, I. Novotný, I. Procházka, F. Šebesta, Nucl. Instr. and Meth. A 443 (2000) 557.

[4] D. Schödlbauer, P. Sperr, G. Kögel, W. Triftshäuser, Nucl. Instr. and Meth. B 34 (1988) 258. 